\title{
Investigation of SU8 as A Structural Material for Fabricating Passive Millimetre-wave and Terahertz Components
}

\author{
Yingtao Tian ${ }^{1,2, *}$, Xiaobang Shang ${ }^{1}$, Yi Wang ${ }^{3}$, Michael J. Lancaster ${ }^{1}$ \\ ${ }^{1}$ School of Electronic, Electrical, and Systems Engineering, University of Birmingham, B15 2TT, UK \\ ${ }^{2}$ School of Materials, University of Manchester, Manchester, M13 9PL, UK \\ ${ }^{3}$ Faculty of Engineering and Sciences, University of Greenwich, Chatham, ME4 4TB, Kent, UK \\ * Author to whom correspondence should be addressed; Email: yingtao.tian@manchester.ac.uk; \\ Tel: $+44(0) 1613063588$
}

\begin{abstract}
This paper is to provide a systematical review of the technical issues of SU8 fabrication for millimeter-wave and terahertz components based on research works carried out at the University of Birmingham in the past decade. A design-for-manufacturability (DFM) approach is followed. The flexibility of the SU8 process enables many novel device structures. Challenges and problems during the fabrication will be discussed and demonstrated with examples. The measurement of the devices is also a significant challenge when the critical dimensions of the device shrink and special testing fixtures are needed in some cases. Finally, a brief overview of the issues discussed above is given for future guidance.
\end{abstract}

Keywords: SU8 photolithography; millimeter-wave and terahertz components

\section{Introduction}

In recent years, there has been an increasing interest in millimeter-wave (mm-wave) and terahertz (THz) components working in the frequency range between $30 \mathrm{GHz}$ and $1 \mathrm{THz}$ due to its widespread potential of applications in near-field security scanning, ultrafast wireless communication, medical imaging and non-destructive characterisation of material properties [1], [2]. However, as the operating frequency increases, the dimensions of the critical features decrease and it becomes more and more challenging for conventional computer numerical controlled (CNC) metal milling to deliver precise components. When it comes to sub-millimeter range (i.e. over $300 \mathrm{GHz}$ ), it is almost impossible to machine out complicated structures required in many waveguide devices, due to the limitation on the physical dimensions of the milling tools. To overcome the limitation of conventional machining, SU8 has been identified as a promising structural material to fabricate mm-wave and $\mathrm{THz}$ components through the photolithography process [3]. SU8 is capable of forming high aspect-ratio 3D submillimeter structures with high dimensional accuracy (with few microns tolerance) and has excellent surface quality. Aspect-ratios as high as 1:50 are achievable through UV exposure and the typical 
surface roughness of SU8 after development is around tens of nanometers. It also offers cost effectiveness and high manufacturing flexibility for the sub-millimeter dimension range, and has a good repeatability at relatively low facility investment.

In the Emerging Device Technologies (EDT) research group at University of Birmingham, a series of passive terahertz devices have been successfully developed using the SU-8 photolithography process. Table 1 lists the details of the passive devices developed in EDT group in the past decade. The devices include rectangular coaxial cables, cavity resonators, rectangular waveguides, filters and antennas working in the frequency range up to $700 \mathrm{GHz}[4]-[28]$. The SU8 fabrication process has demonstrated great capacity to deliver the required structure with high precision and repeatability. In addition to the EDT work, there have been other applications based on SU8 process developed by other research groups in this area. For example, perforated SU8 has been used as the substrate in metamaterials [29]. SU8 has been used as the mold for fabricating copper coil [30]. SU8 was inkjetprinted as the substrate for microwave micro-strips [31]. In [32], microwave resonators have been embedded in multilayer SU8 structures. Thermal bonded SU8 has been investigated for microwave packaging applications [33]. SU8 was also used as ring spacer for polyimide low frequency antenna 2.5-12 GHz [34][35][36]. In other cases, SU8 pillar metamaterials was utilized for THz imaging [37] and SU8 coated with $\mathrm{Cu}$ was employed for coplanar waveguide transmission line at 50GHz [38].

Table 1. Details of passive mm-wave and THz devices developed in EDT group at University of Birmingham

\begin{tabular}{llll}
\hline Device name & Frequency & Features & References (Years) \\
\hline K band rectangular coaxial cable & $14-36 \mathrm{GHz}$ & 5 layers of 300 $\mu \mathrm{m}$ & Ref. [16] (2007) \\
Rectangular coaxial line and cavity & $77 \mathrm{GHz}$ & 5 layers of 200 $\mu \mathrm{m}$ & Ref. [25] (2008) \\
resonator & & & Ref. [23] (2008) \\
Rectangular coaxial cable with bent & $28-48 \mathrm{GHz}$ & 4 layers & \\
supporting stubs & & & Ref. [20] (2009) \\
Rectangular coaxial branch line coupler & $38 \mathrm{GHz}$ & 5 layers of 300 $\mu \mathrm{m}$ & Ref. [6] (2009) \\
Rectangular coaxial line to ridge & $90 \mathrm{GHz}$ & 5 layers of 200 $\mu \mathrm{m}$ & \\
waveguide transition & & & \\
\hline
\end{tabular}




\begin{tabular}{|c|c|c|c|}
\hline $2 \times 2$ Butler matrix with patch array & $63 \mathrm{GHz}$ & 5 layers of $200 \mu \mathrm{m}$ & Ref. [26] (2009) \\
\hline Interdigital filter & $60 \mathrm{GHz}$ & 5 layers of $200 \mu \mathrm{m}$ & Ref. [39] (2009) \\
\hline H-plane horn antenna & $63 \mathrm{GHz}$ & 5 layers of $200 \mu \mathrm{m}$ & Ref. [13] (2010) \\
\hline WR-3 waveguide with right angle bends & $300 \mathrm{GHz}$ & $\begin{array}{l}4 \text { layers of } 432 \mu \mathrm{m} \text {, single } \\
\text { layer and double layer } \\
\text { processes }\end{array}$ & $\begin{array}{l}\text { Ref. [17] (2010), } \\
\text { Ref. [10] (2014) }\end{array}$ \\
\hline WR-3 waveguide with embedded bends & $300 \mathrm{GHz}$ & 4 layers of $432 \mu \mathrm{m}$ & Ref. [22] (2010) \\
\hline Slotted waveguide antenna & $300 \mathrm{GHz}$ & 4 layers $432 \mu \mathrm{m}$. & Ref. [18] (2011) \\
\hline WR 3 band device measurement methods & $300 \mathrm{GHz}$ & $\begin{array}{l}\text { Comparison of measurement } \\
\text { methods }\end{array}$ & Ref. [12] (2011) \\
\hline $\begin{array}{l}\text { W band waveguide and filter with } \\
\text { embedded H-plane bends }\end{array}$ & $88.47 \mathrm{GHz}$ & 6 layers of $635 \mu \mathrm{m}$ & Ref. [27] (2011) \\
\hline U band coaxial filter & $42 \mathrm{GHz}$ & 5 layers of $200 \mu \mathrm{m}$ & Ref. [14] (2011) \\
\hline $\begin{array}{l}\text { WR-3 waveguide filter with embedded } \\
\text { bends }\end{array}$ & $300 \mathrm{GHz}$ & 4 layers of $432 \mu \mathrm{m}$ & Ref. [24] (2011) \\
\hline WR-3 band waveguide diplexer & $300 \mathrm{GHz}$ & 4 layers of various thickness & Ref. [5] (2011) \\
\hline $\begin{array}{l}\text { WR-3 band straight through waveguide, } \\
\text { bandpass filter and dual-band filter }\end{array}$ & $300 \mathrm{GHz}$ & $\begin{array}{l}4 \text { layers of } 432 \mu \mathrm{m} \text {, double } \\
\text { layer process }\end{array}$ & Ref. [21] (2012) \\
\hline Pseudo-elliptical response filter at $\mathrm{W}$ band & $100 \mathrm{GHz}$ & 3 layers $635 \mu \mathrm{m}$ & Ref. [4] (2012) \\
\hline WR 1.5 band waveguide & $700 \mathrm{GHz}$ & 3 layers of $191 \mu \mathrm{m}$ & Ref. [19] (2013) \\
\hline WR-3 band waveguide bandpass filter & $300 \mathrm{GHz}$ & 5 layers of $432 \mu \mathrm{m}$ & Ref. [8] (2013) \\
\hline Thick mesh filter & $300 \mathrm{GHz}$ & $\begin{array}{l}300 \mu \mathrm{m} \text { single layer or } \\
\text { stacked }\end{array}$ & Ref. [28] (2014) \\
\hline Dual-band orthomode transducer & $100 \mathrm{GHz}$ & 6 layers of $635 \mu \mathrm{m}$ & Ref. [11] (2014) \\
\hline
\end{tabular}

Although the SU8 process has been successfully applied in a number of passive devices, the technique is not matured yet and still has room to improve. This paper systematically investigates the technical issues occurring at different stages of the process, i.e. design, fabrication and measurement. The pros 
and cons of this technique will be discussed comparing with the conventional $\mathrm{CNC}$ machining. In the end, guidance for these steps will be given for future development.

\section{Design}

The SU8 process is superior to conventional CNC milling in terms of (i) reduced cost particularly for large scale production; (ii) higher dimensional accuracy; (iii) small side-wall roughness $(<40 \mathrm{~nm})$ and (iv) nearly vertical side wall profile. In order to use SU8 as structural material to form mm-wave devices, the components need to be divided into several slices so that each slice can be fabricated through single or multiple layers of SU8 photolithography. One layer of SU8 represents one segment of the microwave component and the entire device can be formed by assembling all the segments together in the end. It should be noted that the SU8 will be metallised on all surfaces to enable functional microwave devices. This methodology allows taking advantages of SU8 fabrication mentioned above and enables the possibility to realise innovative designs which are not feasible through the CNC machining. Two devices produced using the SU8 process will be given as examples.

The first device is a slotted waveguide antenna operating at WR-3 band (220-325 GHz). It is shown in Figure 1. The waveguide antenna is divided into 4 slices and each slice can be fabricated through one layer of $432 \mu \mathrm{m}$ thick SU8. However, if one wants to use CNC machining to achieve this structure, i.e. to machine out the blue coloured structure shown in Figure 1 a), it would be very challenging due to the 8 delicate radiating slots and the overlapped matching ridges. The second device is a filter operating at WR-1.5 band (500-750 GHz), as shown in Figure 2. It is formed of three SU8 layers each with the same thickness of $191 \mu \mathrm{m}$. The rectangular holes in the middle of each layer offer the filter functionality and are the most crucial parts. These rectangular holes cannot be made by CNC milling because they are offset against each other so that the milling tool cannot reach the central segment. However, by using the SU8 photolithography process, these three layers can be fabricated accurately on one wafer without any restrictions. Both devices have been tested to show good microwave performance. 


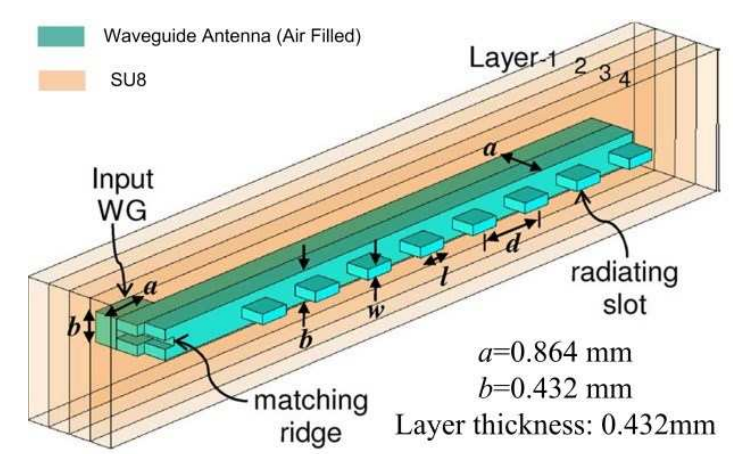

(a)

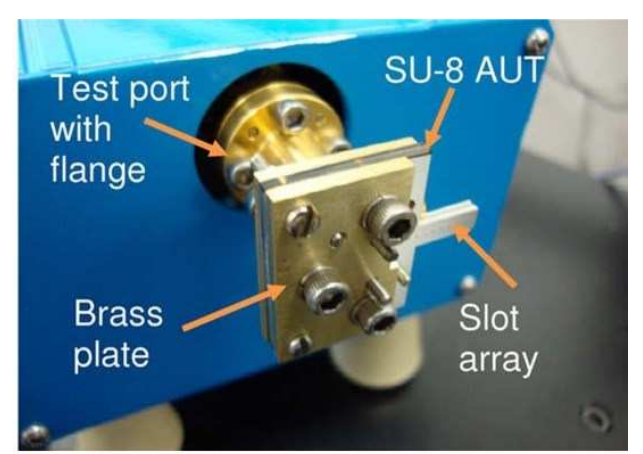

(b)

Figure 1 Diagrams of a WR-3 band antenna [18]. (a) Photograph of the 300-GHz slotted waveguide antenna with the port flange of the test equipment; (b) Enlarged view of the slot array and the H-plane bend formed of four layers (the outstanding structure in the central areas represents the hollow channel and slots).

Like any other techniques, the SU8 process also has limitations. As a guidance of best practice, devices need to be designed to cope with constraints as follows.

1. The design needs to be compatible with the photolithography process. Specifically, the design should not include variable depths in one layer and should incorporate vertical sidewalls only.

2. SU8 is capable of patterning high aspect ratio $(>1: 20)$ structures. In our work, the designs utilize much lower aspect ratios than 1: 20, due to the difficulty of metal coating deep structure using vacuum deposition process (details given in Section 3).

3. A dimensional accuracy of less than $10 \mu \mathrm{m}$ has been demonstrated by the SU8 process developed in our group. This tolerance needs to be born in mind during the design.

4. The single-layer process does not allow isolated islands without support. This problem has been addressed by the later developed two-layer process which enables standalone regions on the top layer [21].

5. The SU8 process is not a desired choice for scenarios where a high thermal stability or a high operating temperature of the devices is required.

6. The SU8 process is capable of producing layers with thickness ranging from $1 \mu \mathrm{m}$ to $2000 \mu \mathrm{m}$. Layer thickness of the design should fall within this range. It is suggested that a layer thickness of above $100 \mu \mathrm{m}$ should be used, to facilitate the handling of the layer. 


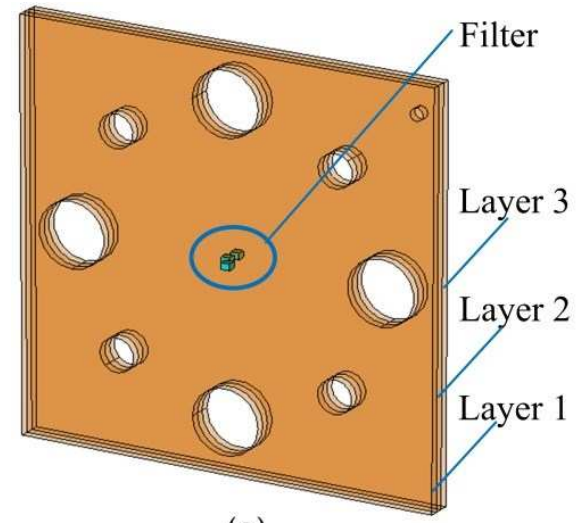

(a)

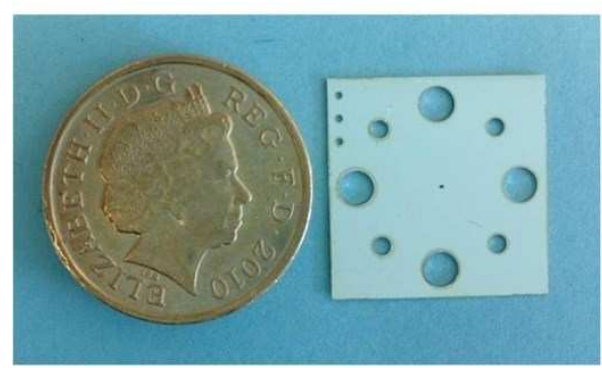

(c)

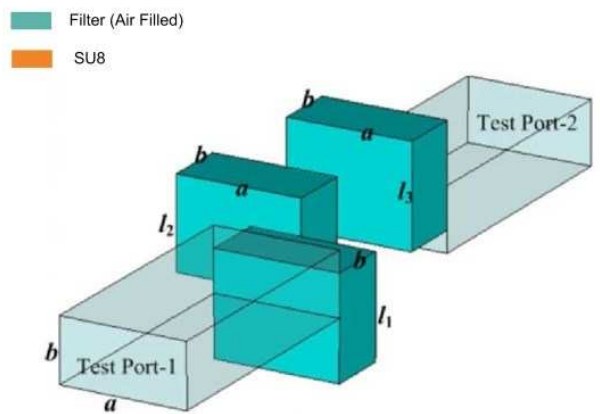

(b)

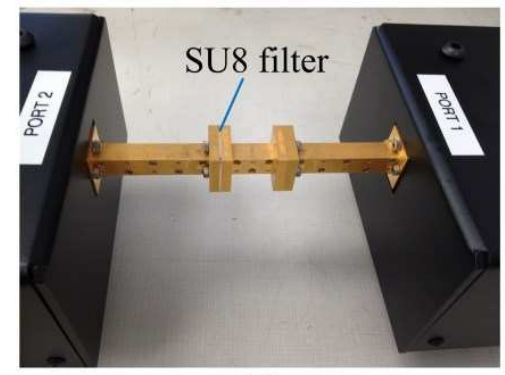

(d)

Figure 2 Diagrams of a WR-1.5 band filter made by SU8 process [9]. (a) Configuration of the whole filter comprising of 3 layers with the same thickness of $191 \mu \mathrm{m}$. The outstanding part stands for the filter. (b) Diagram of the filter structure. (c) Photograph of one SU8 layer with silver coating. (d) Photograph of the measurement setup. The filter (based on three SU8 shims) is sandwiched between two waveguide flanges of measurement equipment.

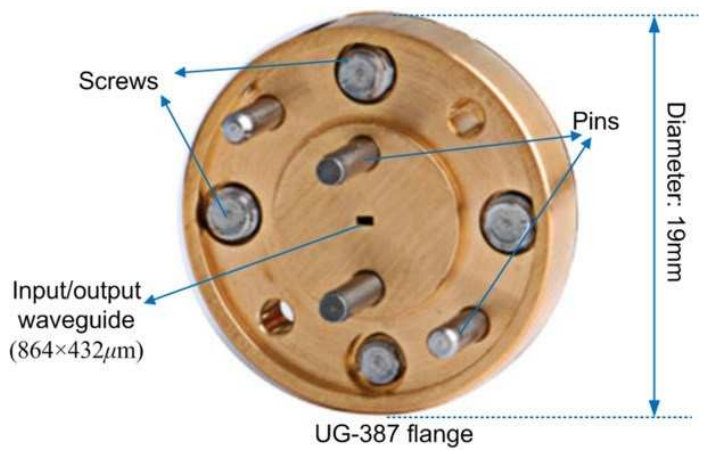

Figure 3 Photograph of a UG-387 flange, which has been used widely by terahertz waveguides.

How to connect the micromachined SU8 devices to the external equipment (e.g. measurement system) should also be considered during the design. Usually waveguide components are interconnected via 
standard flanges and Figure 3 shows an example of such a flange utilised at terahertz frequency bands. As can be observed from|Figure 3, to match with the flange, the size of a SU8 layer should be at least $19 \times 19 \mathrm{~mm}$. SU8 layers with a thickness less than $100 \mu \mathrm{m}$ may be too fragile to handle.

\section{Fabrication}

Depending on the design of the device, the fabrication varies from single layer lithography to doublelayer lithography processes. As demonstrated above, the device is usually divided into several layers to make each layer feasible for SU8 processing. Figure 4 illustrates the typical process flow of the SU8 fabrication which is derived from the standard lithography process. Firstly, SU8 is spin coated on silicon wafer, prebaked and exposed under UV light, i.e. steps (a) and (b) in Figure 4. If the device is divided into several separate single layers, then the (c) step in Figure 4 will form strong crosslink by baking at after exposure and the process stops on step (c) for release, metallization and assembly. If the device has isolated features, which cannot stand alone without a support layer, it will need to go through the full double layer spin coating and crosslink process [21]. Therefore, the first layer will undergo a post exposure bake at relatively lower temperature $\left(70^{\circ} \mathrm{C}\right)$ to form weak crosslink and will not be developed at this stage Figure 4 (d)). The second layer of SU8 will be spin coated on top of the weakly crosslinked first layer and the aforementioned prebake, exposure and post exposure bake will be performed again. It should be noted that the prebake and post exposure bake of double layer SU8 need to be carried out at lower temperature to reduce the stress. For example, the prebake and postbake parameters for the single layered $288 \mu \mathrm{m}$ thick WR-3 band $300 \mathrm{GHz}$ device are $95{ }^{\circ} \mathrm{C}$ for 4.5 hours and 30 mins respectively [22]. For the double layered structure, the prebake for the first layer is same as the single layered process but performed at $70{ }^{\circ} \mathrm{C}$ for much longer time ( 8.5 hours) for the second layer [10]. Once both SU8 layers are strongly crosslinked, the device is ready to be released, metallised and assembled for testing Figure 4(e) - (g)). The double layer process is always preferable because it can reduce the number of inter-layer gaps which could decrease the insertion loss and reduce the risk of device failure. However, this needs to be considered in the design stage to enable the feasibility of the double layer exposure without compromising the performance of the device. It is also worth noting that a thicker wafer is needed as the carrier during the double layer 
process because the stress accumulated in the two layers of SU8 may distort the standard $500 \mu \mathrm{m}$ thick silicon wafer. Moreover, SU8-50 was used in most of our previous studies due to its relatively lower viscosity and cost comparing with other formulas such as SU8-100.

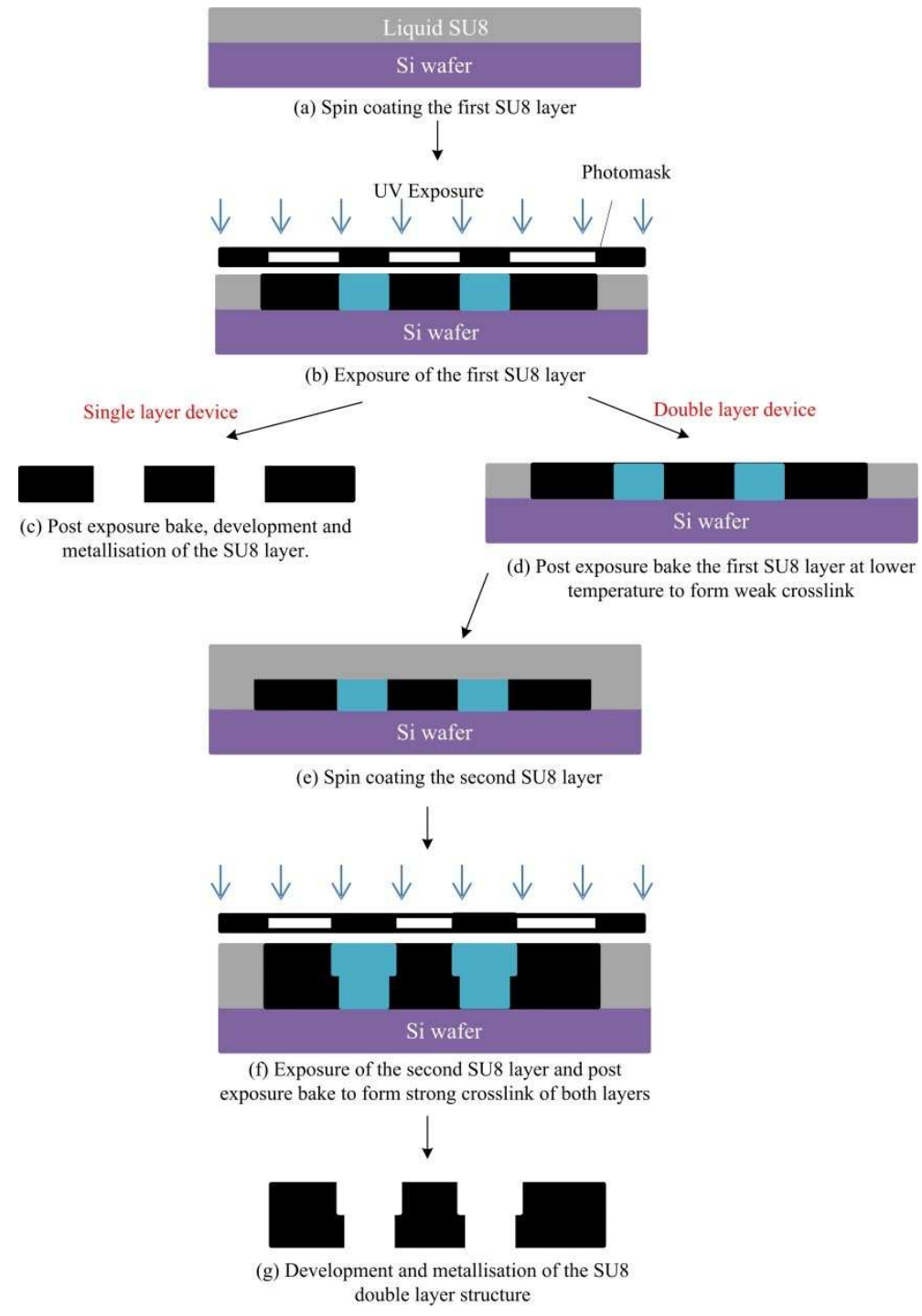

Figure 4 Typical SU8 fabrication process flow

Thickness Control - For the devices designed in the terahertz range, each layer of the SU8 ranges from about $200 \mu \mathrm{m}$ to $600 \mu \mathrm{m}$ and in the case of double layer process the overall thickness can reach nearly $1000 \mu \mathrm{m}$. Because the dimension of the device is designed in proportion to microwave wavelength, small variation on the thickness can have noticeable influences on the microwave performance, e.g. frequency shift. Therefore, it is challenging to process such a thick photoresist in line with the design thickness. The two main factors to be concerned about during the process are 
edge bead removal and SU8 weight control. The edge bead has detrimental effect on the success of the process. When the photoresist is thick, the spin coating parameter becomes less precise and the edge bead needs to be removed with extra care. In addition to the spin coating parameter, the weight of the SU8 on the wafer is also checked and used as a reference to determine the thickness. A series of experiments have been carried out to calibrate the relationship between the weight of SU8 and the final thickness. It has been worked out that 1 gram of liquid SU8 on the $100 \mathrm{~mm}$ diameter wafer correspond to about $83 \mu \mathrm{m}$ in thickness after development. This method can eliminate the uncertainty of how much photoresist is removed from the edge bead each time.

Uniformity - The uniformity of the process measures the within-wafer thickness variation and the wafer-to-wafer consistency. After spin coating and edge bead removal, the wafer needs to rest on a carefully leveled stage to allow the SU8 to achieve self-planarisation. This step is crucial as even a small slope on the hotplate can cause significant error in the final thickness uniformity of the ultrathick SU8 resist [40]. It was found that the thickness uniformity on a single wafer was significantly improved when the wafer was self-planarised for more than 4 hours. The weight checking of the SU8 on each wafer is another way to ensure the uniformity from batch to batch.

Sidewall Angle - The vertical sidewall is another critical aspect to achieve accurate dimensions of the device. Due to the large thickness of the SU8, a special exposure method needs to be adopted to ensure sufficient penetration of UV light and strong enough crosslinking. Depending on the thickness, a L39 filter, which allows light above $400 \mathrm{~nm}$ wavelength to pass through, and a PL360 filter, which allows the $i$-line $365 \mathrm{~nm}$ to pass through, need to be used at various combinations. Another issue affecting sidewall angle is the developing time and process control. To accelerate the photoresist development, the EC solvent developer is heated and agitated by magnetic stirring. Full details of how and when to use this two filter and development process control can be found in the references for each particular device listed in Table 1 .

Metallisation - Once the SU8 pieces are released from the wafer, they need to be metallised with gold or silver to form the conductive guided structures for microwave transmission. As can be seen from 
the design, the sidewalls need to be fully covered with sufficient amount of metal with a thickness of at least 3-5 times of the skin depth. Typically, all the surfaces are coated with $2 \mu \mathrm{m}$ silver or gold, while the skin depth is around $0.2 \mu \mathrm{m}$ at $100 \mathrm{GHz}$ and even smaller at higher frequency, this is enough to ensure a good conductivity. To achieve this, the SU8 pieces are tilted to $30^{\circ}$ angle and rotated throughout the evaporation coating process Figure 5(a)). The aspect ratio of the smallest features in those microwave components are around 1:1 and in theory the maximum tilt angle is about $45^{\circ}$ so that the line-of-sight evaporation will have same efficiency to cover both the sidewall and the flat surface. However, due to the limitation of the equipment, the maximum tilt angle is set as $30^{\circ}$. The line-of-sight evaporation deposits less materials on the sidewall but is still able to cover the all the surfaces of SU8 features. As illustrated in Figure 5 (b) and (c), the SU8 component is flipped over so that both sides are coated with silver. In this case, the sidewall is coated twice to achieve a better uniformity throughout. If the microwave features have larger aspect ratios, calculation of the tilt angle needs to be performed to ensure the line-of-sight evaporation could sufficiently cover the sidewall, or other metallisation technique (e.g. sputtering) can be used to achieve that. Also, the SU8 surface needs to be cleaned with oxygen plasma and primed with $100 \mathrm{~nm}$ Cr layer for better adhesion. In some cases, when the SU8 piece is relatively thin, e.g. less than $200 \mu \mathrm{m}$ thick, or if the metal source is overheated, it is noticed that the heat from the evaporation can deform the SU8 pieces and cause problems with the assembly and alignment. Therefore extra care should to be taken to regulate the thermal impact to the SU8 pieces.

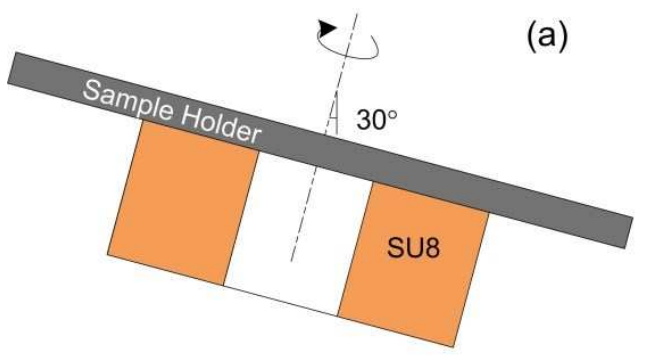

Ag evaporation (b)

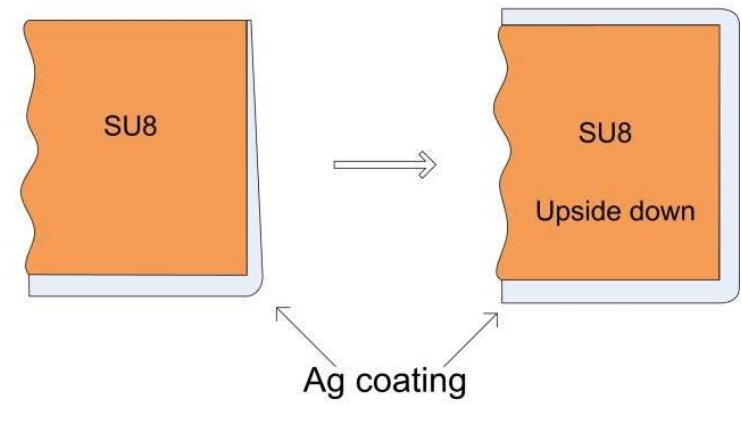

Figure 5 Schematics of metallising the side wall of SU8 components through evaporation: (a) configuration of the SU8 components in the evaporation chamber; (b) silver thickness distribution after coating on one side and (c) full coverage of sidewall with silver after evaporation on both sides. 


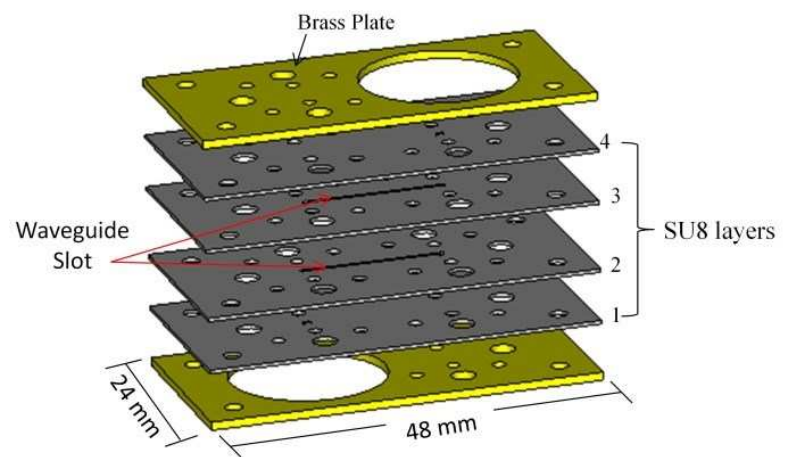

(a)

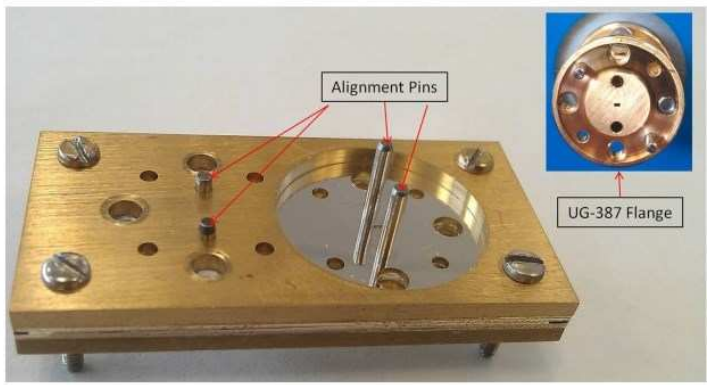

(b)

Figure 6 Typical integration structure of passive terahertz device [10].

Integration - The metallised SU8 pieces are still fragile and easy to break, so a brass bracket is usually used to protect the device and to facilitate connection to the measurement kit. Figure 6 shows the typical device packing method. To assemble the metallised SU8 pieces into a functional device, a number of alignment pins, which have very accurate dimensions $( \pm 20 \mu \mathrm{m})$, are used to ensure all the features in different layers are aligned precisely. This alignment pins are also used as dwell pins to fit with the waveguide flanges on the measurement port. Because the brass bracket is usually fastened by four screws in each corner, it is also very important to ensure all the corners are tightened to same extent.

\section{Measurement}

\subsection{Dimensional measurement of the device during fabrication process}

For the multi-stage fabrication process, it is worth to measure the dimensions of the SU8 pieces at different stages of the process in order to confirm the fore-end process was performed on a satisfactory level and the dimensions are within acceptable tolerance. There are three important measurements to be carried out before the assembly of the device. First, after the SU8 is developed on the wafer, an optical microscope is used to examine whether the features and the dimensions of the critical apertures are developed properly Figure 7 (a)). Second, it is important to measure the thickness of SU8 layer and determine the uniformity across wafer. White light interferometer is used in this step as it offers a convenient, accurate, non-contact and fast measurement. If the thickness of SU8 is found out of the tolerable range, the sample will be abandoned and the measured data will 
feedback to adjust the previous fabrication parameters. Third, once the SU8 layer is fully coated with metal, the final dimensions of the microwave transmission slots and apertures are recorded using a good optical microscope. We also inspect any damage to the device Figure 7 (b)). Scanning electron microscope (SEM) is usually utilized to check the quality of surface metallization and the integrity of the double layers Figure 7 (c)).

(a)

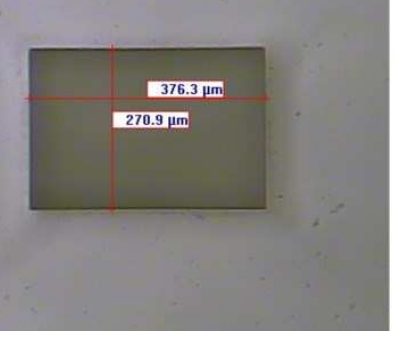

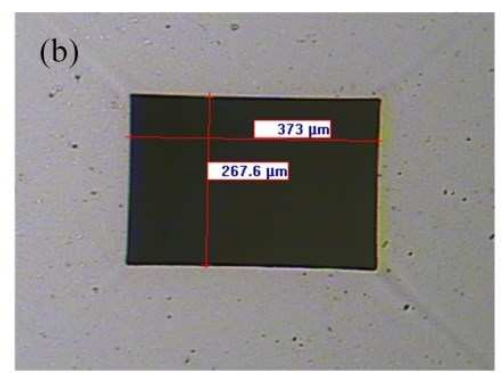

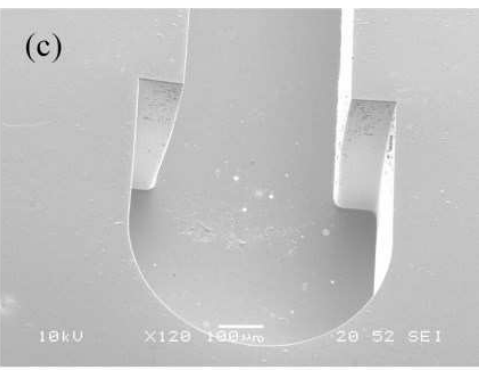

Figure 7 Measurement of SU8 pieces at different stages of the fabrication process: (a) after development; (b) after metallization; and (c) metallization quality and double layer integrity inspection in SEM.

\subsection{Measurement of the packaged devices}

To characterize the microwave performance of these packaged devices, two sets of calibrated microwave measurement methods are usually utilized, as follows

i. For coaxial devices, as discussed in [20], they are tested with an on-wafer probe station using a pair of ground-signal-ground probes.

ii. For waveguide and filter devices, either embedded bends or test fixture are conventionally made from copper (e.g. [10], [21]). This is explained in detail in [17]. Additionally, direct connection with measurement equipment is utilized for filters with a particular type of topology (e.g. [9]). Such connection configuration eliminates the influence from the text fixture and ultimately yields a low transmission loss.

The transmission loss, also termed as insertion loss (IL), is commonly used to indicate the microwave signal attenuation through the device. Lower IL presents better performance. The measurement results 
of the WR-1.5 band filter (see Figure 2, are shown in Figure 8, which exhibits a $3 \mathrm{~dB}$ bandwidth of $53.7 \mathrm{GHz}$ at a centre frequency of $671 \mathrm{GHz}$. The median passband insertion loss is measured to be $0.65 \mathrm{~dB}$, which is close to the theoretical value of $0.28 \mathrm{~dB}$ acquired from simulations. These are excellent results and this filter is one of a very few demonstrated at this frequency band across the world. The WR-3 band antenna (see Figure 1) is also measured to have an excellent performance, as shown in Figure 9. The measured normalized H-plane radiation pattern agrees very well with the simulation. This indicates good dimensional accuracy for the radiation slots, rendered by the lithography-based fabrication process.

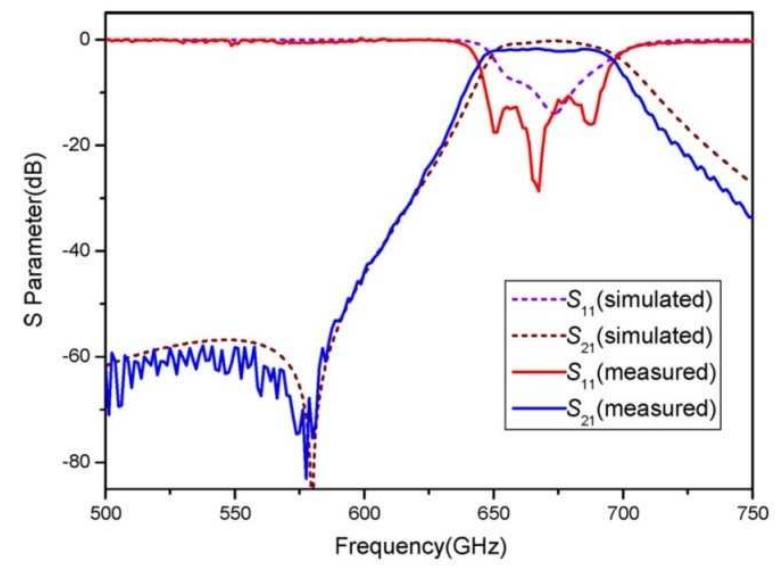

Figure 8 Measurement results of the WR1.5 band filter made from SU8 [9].

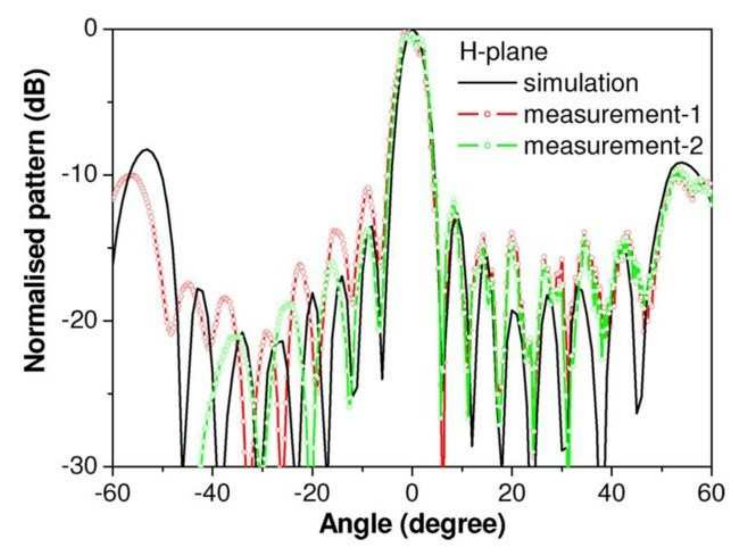

Figure 9 Measurement results of the WR-3 antenna [18], measured H-plane radiation patterns in comparison to simulations. 


\section{Summary}

In summary, the design, fabrication, packaging and testing of mm-wave and $\mathrm{THz}$ passive devices have been reviewed on the basis of a series of devices developed through the SU8 processing at the University of Birmingham. Challenges and problems of using SU8 as a main structural material for fabricating sub-millimeter wave components have been discussed. The excellent test results have shown that the SU8 process provides a competitive solution for the next generation Terahertz communication system. The following points can be concluded and referenced for future studies. Firstly, the design of the devices needs to consider manufacturing process. Also, the advantages of SU8 process enable novel structures which are not possible to achieve using conventional CNC machining. Secondly, the fabrication process is outside the standard SU8 photolithography working window and additional approaches are needed to achieve high accuracy and reproducibility. Finally, the packaging of the devices needs to meet the requirement of the test fixtures which may only allow certain types of methods and techniques.

\section{References:}

[1] I. Hosako, N. Sekine, M. Patrashin, S. Saito, K. Fukunaga, Y. Kasai, P. Baron, T. Seta, J. Mendrok, S. Ochiai, and H. Yasuda, "At the Dawn of a New Era in Terahertz Technology," Proc. IEEE, vol. 95, no. 8, pp. 1611-1623, Aug. 2007.

[2] D. M. Sheen, D. L. McMakin, and T. E. Hall, "Near Field Imaging at Microwave and Millimeter Wave Frequencies," in 2007 IEEE/MTT-S International Microwave Symposium, 2007, pp. 1693-1696.

[3] C. M. Mann, "Fabrication technologies for terahertz waveguide," in 1998 IEEE Sixth International Conference on Terahertz Electronics Proceedings. THZ 98. (Cat. No.98EX171), 1998, pp. 46-49.

[4] C. A. Leal-Sevillano, J. R. Montejo-Garai, M. Ke, M. J. Lancaster, J. A. Ruiz-Cruz, and J. M. Rebollar, "A Pseudo-Elliptical Response Filter at W-Band Fabricated With Thick SU-8 PhotoResist Technology," IEEE Microw. Wirel. Components Lett., vol. 22, no. 3, pp. 105-107, Mar. 2012.

[5] T. Skaik, M. Lancaster, Maolong Ke, and Yi Wang, "A micromachined WR-3 band waveguide diplexer based on coupled resonator structures," in 201141 st European Microwave Conference (EuMC), 2011, pp. 770-773.

[6] N. A. Murad, M. J. Lancaster, Y. Wang, and M. L. Ke, "Micromachined rectangular coaxial 
line to ridge waveguide transition," in 2009 IEEE 10th Annual Wireless and Microwave Technology Conference, 2009, pp. 1-5.

[7] Y. Wang and M. J. Lancaster, "A micromachined centre-fed slotted waveguide antenna for mm-wave applications," in 2012 IEEE MTT-S International Microwave Workshop Series on Millimeter Wave Wireless Technology and Applications, 2012, pp. 1-3.

[8] J. Xu, Y. Tian, X. Shang, Q. Chen, and M. J. Lancaster, "SU-8 micromachined WR-3 band waveguide bandpass filter with low insertion loss," Electron. Lett., vol. 49, no. 7, pp. 480-482, Mar. 2013.

[9] X. Shang, Y. Tian, M. J. Lancaster, and S. Singh, "A SU8 Micromachined WR-1.5 Band Waveguide Filter," IEEE Microw. Wirel. Components Lett., vol. 23, no. 6, pp. 300-302, Jun. 2013.

[10] Y. Tian, X. Shang, and M. J. Lancaster, "Fabrication of multilayered SU8 structure for terahertz waveguide with ultralow transmission loss," J. Micro/Nanolithography, MEMS, MOEMS, vol. 13, no. 1, p. 013002, Jan. 2014.

[11] C. A. Leal-Sevillano, Y. Tian, M. J. Lancaster, J. A. Ruiz-Cruz, J. R. Montejo-Garai, and J. M. Rebollar, "A Micromachined Dual-Band Orthomode Transducer," IEEE Trans. Microw. Theory Tech., vol. 62, no. 1, pp. 55-63, Jan. 2014.

[12] Y. Wang, M. J. Lancaster, M. Ke, and X. Shang, "Measurements of micromachined waveguide devices at WR-3 band using a T/R-T module based network analyzer," in 77 th ARFTG Microwave Measurement Conference, 2011, pp. 1-4.

[13] N. A. Murad, M. J. Lancaster, P. Gardner, M. L. Ke, and Y. Wang, "Micromachined H-plane horn antenna manufactured using thick SU-8 photoresist," Electron. Lett., vol. 46, no. 11, p. $743,2010$.

[14] A. Jaimes-Vera, I. Llamas-Garro, M. Ke, Y. Wang, M. J. Lancaster, and L. Pradell, "U-band micromachined coaxial filter," in 2011 IEEE MTT-S International Microwave Workshop Series on Millimeter Wave Integration Technologies, 2011, pp. 184-187.

[15] Y. Wang, M. Ke, and M. J. Lancaster, "Micromachined $38 \mathrm{GHz}$ cavity resonator and filter with rectangular-coaxial feed-lines," IET Microwaves, Antennas Propag., vol. 3, no. 1, p. 125, 2009.

[16] M. J. Lancaster, J. Zhou, M. Ke, Y. Wang, and K. Jiang, "Design and High Performance of a Micromachined K-Band Rectangular Coaxial Cable," IEEE Trans. Microw. Theory Tech., vol. 55, no. 7, pp. 1548-1553, Jul. 2007.

[17] X. Shang, M. J. Lancaster, M. Ke, and Y. Wang, "Measurements of micromachined submillimeter waveguide circuits," in 2010 76th ARFTG Microwave Measurement Conference, 2010 , pp. $1-4$. 
[18] Y. Wang, M. Ke, and M. J. Lancaster, "Micromachined 300-GHz SU-8-Based Slotted Waveguide Antenna," IEEE Antennas Wirel. Propag. Lett., vol. 10, pp. 573-576, 2011.

[19] X. Shang, Y. Tian, M. J. Lancaster, and S. Singh, "A SU8 Micromachined WR-1.5 Band Waveguide Filter," IEEE Microw. Wirel. Components Lett., vol. 23, no. 6, pp. 300-302, Jun. 2013.

[20] Y. Wang, M. Ke, M. J. Lancaster, and F. Huang, "Micromachined Millimeter-Wave Rectangular-Coaxial Branch-Line Coupler With Enhanced Bandwidth," IEEE Trans. Microw. Theory Tech., vol. 57, no. 7, pp. 1655-1660, Jul. 2009.

[21] X. Shang, M. Ke, Y. Wang, and M. J. Lancaster, "WR-3 Band Waveguides and Filters Fabricated Using SU8 Photoresist Micromachining Technology," IEEE Trans. Terahertz Sci. Technol., vol. 2, no. 6, pp. 629-637, Nov. 2012.

[22] T. Skaik, Yi Wang, Maolong Ke, Shilong Qian, and M. Lancaster, "A micromachined WR-3 waveguide with embedded bends for direct flange connections." pp. 1225-1228, 2010.

[23] M. Ke, Y. Wang, and M. Lancaster, "Design and realisation of low loss air-filled rectangular coaxial cable with bent quarter-wavelength supporting stubs," Microw. Opt. Technol. Lett., vol. 50, no. 5, pp. 1443-1446, May 2008.

[24] X. Shang, M. L. Ke, Y. Wang, and M. J. Lancaster, "Micromachined WR-3 waveguide filter with embedded bends," Electron. Lett., vol. 47, no. 9, p. 545, 2011.

[25] M. Ke, Y. Wang, K. Jiang, and M. J. Lancaster, "Micromachined rectangular coaxial line and cavity resonator for $77 \mathrm{GHz}$ applications using SU8 photoresist," in 2008 Asia-Pacific Microwave Conference, 2008, pp. 1-4.

[26] N. A. Murad, M. J. Lancaster, Y. Wang, and M. L. Ke, "Micromachined millimeter-wave Butler matrix with a patch antenna array," in 2009 Mediterrannean Microwave Symposium (MMS), 2009, pp. 1-4.

[27] X. Shang, M. Ke, Y. Wang, and M. J. Lancaster, "Micromachined W-band waveguide and filter with two embedded H-plane bends," IET Microwaves, Antennas Propag., vol. 5, no. 3, p. 334, 2011.

[28] Y. Wang, B. Yang, Y. Tian, R. S. Donnan, and M. J. Lancaster, "Micromachined Thick Mesh Filters for Millimeter-Wave and Terahertz Applications," IEEE Trans. Terahertz Sci. Technol., vol. 4, no. 2, pp. 247-253, Mar. 2014.

[29] I. E. Khodasevych, G. Kostovski, W. S. T. Rowe, and A. Mitchell, "Nonlinear microwave metamaterial resonators using gravitational restoring force realized on a microfabricated perforated substrate," Appl. Phys. Lett., vol. 105, no. 18, p. 181908, Nov. 2014. 
[30] N. Dimitrakopoulos, R. E. Miles, and J. Corda, "Electromagnetically actuated MEMS microwave switch," in 2010 IEEE International Conference on Industrial Technology, 2010, pp. 495-499.

[31] B. K. Tehrani, J. Bito, B. S. Cook, and M. M. Tentzeris, "Fully inkjet-printed multilayer microstrip and T-resonator structures for the RF characterization of printable materials and interconnects," in 2014 IEEE MTT-S International Microwave Symposium (IMS2014), 2014, pp. 1-4.

[32] X. Cheng, P. Jao, C. Kim, and Y. K. Yoon, "Compact 3D integrable SU8 embedded microwave bandpass filters using complementary split ring resonator loaded half mode substrate integrated waveguide," in 2011 IEEE 61st Electronic Components and Technology Conference (ECTC), 2011, pp. 1963-1969.

[33] S. R. Kuppireddi, S. Pamidighantam, V. Janardhana, O. Sorasen, J. S. Roy, and R. G. Kulkarn, "Evaluation of SU8 photo polymer for microwave packaging applications," in NORCHIP 2012, 2012, pp. 1-4.

[34] S. K. Pavuluri, C. H. Wang, and A. J. Sangster, "A High-Performance Aperture-Coupled Patch Antenna Supported by a Micromachined Polymer Ring," IEEE Antennas Wirel. Propag. Lett., vol. 7, pp. 283-286, 2008.

[35] C. H. Wang and S. K. Pavuluri, "Fabrication and assembly of high gain MEMS antennas for wireless communications," in 2008 58th Electronic Components and Technology Conference, 2008, pp. 1941-1945.

[36] S. K. Pavuluri and A. J. Sangster, "High Efficiency Wideband Aperture-Coupled Stacked Patch Antennas Assembled Using Millimeter Thick Micromachined Polymer Structures," IEEE Trans. Antennas Propag., vol. 58, no. 11, pp. 3616-3621, Nov. 2010.

[37] A. J. Gallant, D. Wood, J. A. Levitt, M. Kaliteevski, M. C. Petty, S. Brand, R. A. Abram, and J. M. Chamberlain, "The Fabrication of High Aspect Ratio THz Metamaterials." pp. 117-120, 2006.

[38] F. D. Mbairi and H. Hesselbom, "High frequency design and characterization of SU-8 based conductor backed coplanar waveguide transmission lines," in Proceedings. International Symposium on Advanced Packaging Materials: Processes, Properties and Interfaces, 2005. 2005, pp. 243-248.

[39] Y. Wang, M. Ke, and M. J. Lancaster, "Micromachined 60GHz Air-filled Interdigital Bandpass Filter," in International Workshop on Microwave Filters, 2009.

[40] J. D. Williams and W. Wang, "Study on the postbaking process and the effects on UV lithography of high aspect ratio SU-8 microstructures," J. Micro/Nanolithography, MEMS, MOEMS, vol. 3, no. 4, pp. 563-568, 2004. 


\section{Figure Caption List}

Figure 1 Diagrams of a WR-3 band antenna [18]. (a) Photograph of the 300-GHz slotted waveguide antenna with the port flange of the test equipment; (b) Enlarged view of the slot array and the H-plane bend formed of four layers (the outstanding structure in the central areas represents the hollow channel and slots).

Figure 2 Diagrams of a WR-1.5 band filter made by SU8 process [9]. (a) Configuration of the whole filter comprising of 3 layers with the same thickness of $191 \mu \mathrm{m}$. The outstanding part stands for the filter. (b) Diagram of the filter structure. (c) Photograph of one SU8 layer with silver coating. (d) Photograph of the measurement setup. The filter (based on three SU8 shims) is sandwiched between two waveguide flanges of measurement equipment.

Figure 3 Photograph of a UG-387 flange, which has been used widely by terahertz waveguides.

Figure 4 Typical SU8 fabrication process flow

Figure 5 Schematics of metallising the side wall of SU8 components through evaporation: (a) configuration of the SU8 components in the evaporation chamber; (b) silver thickness distribution after coating on one side and (c) full coverage of sidewall with silver after evaporation on both sides.

Figure 6 Typical integration structure of passive terahertz device [10].

Figure 7 Measurement of SU8 pieces at different stages of the fabrication process: (a) after development; (b) after metallization; and (c) metallization quality and double layer integrity inspection in SEM.

Figure 8 Measurement results of the WR1.5 band filter made from SU8 [9].

Figure 9 Measurement results of the WR-3 antenna [18], measured H-plane radiation patterns in comparison to simulations.

\section{Authors Biographies:}

Yingtao Tian received his $\mathrm{PhD}$ in Mechanical Engineering from Loughborough University, UK, in 2010. His research focused on miniaturization of microelectronics, advanced electrochemical fabrication and precise manufacturing. After two years research in fabrication of terahertz microwave circuits in University of Birmingham, he is currently a Research Associate with the School of Materials in University of Manchester. He is present research interests are advanced laser processing and additive manufacturing for aerospace applications.

Xiaobang Shang received the Ph.D. degree in microwave engineering from the University of Birmingham in 2011. He is currently a Research Fellow with the School of Electronic, Electrical, and Computer Engineering, the University of Birmingham, U.K. His present main research interests are laser micromachined millimeter-wave and sub-millimeter-wave waveguide circuits, mode-matching 
technique for waveguide components, the design of multi-band filters and multiplexers and the integration of antenna and filter.

Yi Wang received his Ph.D. degree in Electronic and Electrical Engineering from The University of Birmingham in 2005. He started his career as a Research Fellow in 2004 at The University of Birmingham, and became a Senior Lecturer at the University of Greenwich in 2011. His present research interests include millimeter-wave / terahertz devices for metrology, communications and sensors, microwave circuits based on multi-port filtering networks, and antennas.

Prof. Michael J. Lancaster is Fellow of the IET, UK, and the UK Institute of Physics. He is a Chartered Engineer in the U.K., and Chartered Physicist in the UK. He has served on the IEEE MTT IMS technical committee. His present research interests include microwave filters and antennas, the high frequency properties and applications of a number of novel and diverse materials. He has published two books and over 170 papers in refereed journals. 